HOMICIDE

\title{
Homicide of children aged 0-4 years, 2003-04: results from the National Violent Death Reporting System
}

\author{
M D Bennett Jr, J Hall, L Frazier Jr, N Patel, L Barker, K Shaw
}

Injury Prevention 2006;12(Suppl II):ii39-ii43. doi: 10.1136/ip.2006.012658

See end of article for authors' affiliations

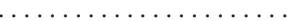

Correspondence to: DrM D Bennett, Jr, Centers for Disease Control and

Prevention, National

Center for Injury

Prevention and Control 4770 Buford Highway, NE

MS K-60, Atlanta, GA

30341-3724, USA;

MBennettJr@cdc.gov

Accepted

7 September 2006

\begin{abstract}
Introduction: To better understand, and ultimately prevent, infant/child homicide, it is imperative to more thoroughly elucidate the circumstances and conditions related to such instances. Data were obtained from the US National Violent Death Reporting System (NVDRS) to illuminate circumstances related to homicide among children aged 4 years or less, and to identify demographic groups which may be at increased risk. Methods: The NVDRS is an active surveillance system that provides comprehensive information on all violent deaths that occur within participating states within the US. Standard statistical tests were conducted to determine homicide rates among children ages 0-4 across states that provided data for both 2003 and 2004 (Alaska, Maryland, Massachusetts, New Jersey, Oregon, South Carolina, and Virginia). These data were further used to investigate infant/child homicides by race, gender, and other relevant circumstances (for example, victim-suspect relationship, weapon type, and location of homicide). A Poisson regression model was fitted to the sample data to investigate the multivariate relationship between the infant/child homicide rate and available demographic information.

Results: The 2003 homicide rate for children ages $0-4$ was 3.0 per 100000 population. The 2004 homicide rate was 2.5 per 100000 population. African Americans were 4.2 times as likely as whites to be victims of homicide. Suspects were commonly parents/caregivers. The vast majority of infant/child homicides occurred in houses or apartments, using weapons that include household objects.

Conclusion: Homicides of infants and young children are most often committed in the home, by parents/ caregivers, using "weapons of opportunity". This suggests that the risk of infant/child homicide is greatest within the primary care giving environment. Moreover, the use of "weapons of opportunity" may be indicative of maladaptive stress responses. Prevention and intervention strategies to reduce infant/child homicide should target the home environment and attend to maladaptive stress responses.
\end{abstract}

$\mathrm{H}$ omicide is the leading cause of infant deaths due to intentional injury in the US. ${ }^{2}$ In 2003, there were 717 reported homicides of children between zero and four years of age. ${ }^{1{ }^{3} 4}$ The risk for homicide is greater in infancy than in any other year of childhood before the age of 15 years. ${ }^{3}$

Prior research suggests that the vast majority of perpetrators of infant/child homicide are female, most often the mother. ${ }^{2}$ As such, certain maternal characteristics have been cited as well established risk factors for infant/child homicide: age 19 or younger; single marital status; 12 or fewer years of education; previous births; and late initiation of prenatal care. ${ }^{35-7}$ Further, infant characteristics associated with infant homicide include low birth weight, low gestational age, low Apgar scores, and male sex. ${ }^{8-10}$

Using data obtained from the National Violent Death Reporting System (NVDRS), the current study explores the circumstances and characteristics of homicide among children aged four years or less.

\section{METHODS}

\section{The NVDRS}

The NVDRS is an active state based surveillance system that provides comprehensive information on all violent deaths within participating states within the US. Seven states (Alaska, Maryland, Massachusetts, New Jersey, Oregon, South Carolina, and Virginia) provided data to the NVDRS in 2003. Six additional states (Colorado, Georgia, North Carolina, Oklahoma, Rhode Island, and Wisconsin) provided data to the NVDRS in 2004. In the current study, we report only on the seven states that began providing data in 2003.

The NVDRS defines violent death as death resulting from an intentional act (for example, poisoning) and/or the intentional use of physical force or power against oneself or another person. This includes homicides, suicides, and legal intervention deaths. Unintentional firearm related deaths and deaths of undetermined intent are also included in the NVDRS. Legal executions and acts of war are excluded. The primary data sources for the NVDRS are death certificates, coroner/medical examiner records, police records, and crime lab data. Secondary, optional data sources include child fatality review team findings, supplementary homicide reports, hospital records, emergency department data, and Bureau of Alcohol, Tobacco, Firearms and Explosives (ATF) trace information on firearms. The NVDRS makes a unique contribution to injury surveillance in that information on circumstances related to suicide, undetermined deaths, homicides, and unintentional firearm injuries are contained in a single database.

\section{Statistical methods}

We determined the homicide rate among children aged four years and under across participating states. In addition, infant/child homicide rates were stratified by race, gender, and other relevant circumstances and conditions (for example, victim-suspect relationship, weapon type) to enable examination of stratum specific variations in rates. Further, we fit a Poisson regression model to investigate the multivariate relationship between the infant/child homicide rate and available demographic information and to facilitate simultaneous statistical adjustment for the effects of all variables of interest. Denominator data were obtained from

Abbreviations: CFR, Child Fatality Review; NVDRS, National Violent Death Reporting System. 
the National Center for Health Statistics. ${ }^{11}$ We determined the percentage of homicides with particular suspect-victim relationships, weapons, and location of homicide. To incorporate into the estimation process an allowance for random variability in the observations, 95\% confidence intervals (CIs) were generated relative to all percentages and parameter estimates. CIs for rates were calculated using asymptotic methods. ${ }^{12} 13$

\section{RESULTS}

We report homicide rates, victim-suspect relationship, weapons used in homicide, and location and timing of homicides.

\section{Homicide rates}

During the two year period spanning 2003 and 2004 the NVDRS recorded a total of 129 deaths of children aged four years or less across the seven states that reported data in both 2003 and 2004. Seventy deaths occurred in 2003; the remaining 59 deaths occurred in 2004 (see table 1). This translates to 3.0 deaths per 100000 population (95\% CI 2.3 to 3.7 ) in 2003 and 2.5 deaths per 100000 population (95\% CI 1.9 to 3.2 ) in 2004. Overall, the infant/child homicide rates for 2003 and 2004 were not significantly different $(p=0.28)$.

In both 2003 and 2004, African Americans accounted for $51 \%$ of homicide victims age four years or less. As shown in table 1, the rate per 100000 population for African American children in 2003 was 7.2 (95\% CI 5.2 to 9.9) and 5.8 (95\% CI 4.1 to 8.3$)$ in 2004. The rates per 100000 population for males in 2003 was 3.5 (95\% CI 2.6 to 4.7 ) and 2.2 (95\% CI 1.5 to 3.2 ) in 2004. The corresponding rates for females were 2.4 (95\% CI 1.7 to 3.5 ) in 2003 and 2.7 (95\% CI 1.9 to 3.8 ) in 2004.

To investigate the multivariable relationship among homicide rate and certain demographic characteristics, we fit a Poisson regression model, with number of homicides as the dependent variable and, as independent variables, year of report (2003 (reference level), 2004), gender (male (reference level), female), race (African American (reference level), white, and other), and all two-way interactions. All multivariate analyses also accounted for state-specific variations in infant homicide rates by treating state as a seven-level factor in the Poisson regression models. We used backwards stepwise regression to identify statistically significant variables.

In the multivariate model, only race and the factor representing state-specific variations in infant homicide rates remained after stepwise regression. Controlling for the effects of other factors, African American infants were 4.2 times

Table 1 Number of deaths for children ages 0-4 recorded in the NVDRS, 2003-04, overall, by race*

\begin{tabular}{llll} 
& Year & Deaths & $\begin{array}{l}\text { Rate per } 100000 \\
\text { population }(95 \% \mathrm{CI})\end{array}$ \\
\hline Overall & 2003 & 70 & $3.0(2.3$ to 3.7$)$ \\
Whites & 2004 & 59 & $2.4(1.9$ to 3.2$)$ \\
& 2003 & 32 & $1.9(1.3$ to 2.6$)$ \\
African Americans & 2004 & 23 & $1.3(0.9$ to 2.0$)$ \\
All other races & 2003 & 36 & $7.2(5.2$ to 9.9$)$ \\
& 2004 & 30 & $5.8(4.1-8.3)$ \\
Males & 2003 & 2 & $\dagger$ \\
& 2004 & 6 & $\dagger$ \\
Females & 2003 & 42 & $3.5(2.6$ to 4.7$)$ \\
& 2004 & 27 & $2.2(1.5$ to 3.2$)$ \\
& 2003 & 28 & $2.4(1.7$ to 3.5$)$ \\
& 2004 & 32 & $2.7(1.9$ to 3.8$)$ \\
\hline
\end{tabular}

*Presented figures are for Alaska, Maryland, Massachusetts, New Jersey, Oregon, South Carolina, and Virginia.

†Rates not reported due to small cell sizes.
(95\% CI 2.9 to 6.0 ) times more likely be homicide victims than white infants. Previous studies have found that male gender is a risk factor. If we do not control for state, gender effects are significant. State specific variation in homicide might be a confounder of the association between gender and homicide victimization.

\section{Victim-suspect relationship}

We also explored the victim-suspect relationships for homicides of children aged four years or less by race and gender for 2003-04. Parents and other caregivers were most often the primary suspects, followed by family acquaintances and friends. Parents and other caregivers were identified as the primary suspect(s) in 52\% of homicides involving African American female infants and young children, and $49 \%$ of homicides involving African American male infants and young children. In comparison, parents were identified as the primary suspect in $50 \%$ of homicides involving white male infants and young children, and 39\% of homicides involving white female infants and young children (see table 2). Moreover, the overall rate of homicides by parents/guardians for 2003-04 was 3.3 per 100000 population (95\% CI 2.8 to 3.9).

While the percentage of homicides in which parents or other caregivers were suspects was similar across most of the comparison groups (white females were the exception, displaying the only percentage that did not approach or surpass $50 \%$ ), comparisons of homicide rates did however reveal that the risk of being killed by a parent or other caregiver was greater for African Americans than for whites. The risk of an African American male infant/child dying in a homicide for which a parent or other caregiver was the suspect was 3.4 times (95\% CI 1.7 to 6.8 ) greater than the risk for white males and 5.8 times greater than the risk for white females (95\% CI 2.6 to 13.1). The largest relative risks for homicide by parent(s) were observed among African American females. Compared to white males and females, the risks of being killed in a homicide in which a parent or other caregiver was suspect were 3.8 (95\% CI 1.9 to 7.4 ) and 6.4 times (95\% CI 2.8 to 14.3) greater for these children.

Family acquaintances and friends were also suspects in a sizeable portion of homicide deaths among those aged 0-4 years. Family acquaintances and friends were identified as suspects in $30 \%$ of homicide deaths involving white female infants/children, and $28 \%$ of homicide deaths involving white male infants/children. In comparison, family acquaintances and friends were identified as suspects in $24 \%$ of homicides involving African American female infants and young children, and $12 \%$ of homicide deaths involving African American male infants and young children.

We found no significant differences in the rates of homicides in which acquaintances/friends were suspects for African American male, white male, and white female infants/children. However, our findings indicate that African American females were 3.9 times more likely to die in a homicide in which a family acquaintance/friend was a suspect than were white females (95\% CI 1.4 to 10.6) and 3.2 times more likely than white males (95\% CI 1.2 to 8.2 ).

\section{Weapon type}

The NVDRS weapon-type categories include firearms, blunt instruments (for example, club or bat), sharp instruments (for example, knife, razor, broken glass, etc), poisoning, hanging (including strangulation and suffocation), personal weapons (for example, fist, feet, hands). Weapons not corresponding to any of the aforementioned categories were designated "other." In 2003-04 "other" weapons constituted the most common weapon type for homicide perpetration. Weapons designated as "other" were cited in 32\% of 
Table 2 Select incident characteristics: frequency and percentage by race and sex*

\begin{tabular}{|c|c|c|c|c|c|c|c|c|c|c|c|c|}
\hline \multirow[b]{3}{*}{ Characteristic } & \multicolumn{4}{|l|}{ White } & \multicolumn{4}{|c|}{ African American } & \multicolumn{4}{|c|}{ Other } \\
\hline & \multicolumn{2}{|l|}{ Males } & \multicolumn{2}{|c|}{ Females } & \multicolumn{2}{|l|}{ Males } & \multicolumn{2}{|c|}{ Females } & \multicolumn{2}{|c|}{ Males } & \multicolumn{2}{|c|}{ Females } \\
\hline & $n(32)$ & $\%$ & $n(23)$ & $\%$ & $n(33)$ & $\%$ & $n(33)$ & $\%$ & $n(4)$ & $\%$ & $n(4)$ & $\%$ \\
\hline \multicolumn{13}{|l|}{ Victim-suspect relationship } \\
\hline Parent & 16 & 50 & 9 & 39 & 16 & 48 & 17 & 52 & 2 & 50 & - & - \\
\hline Other relative & 1 & 3 & - & - & 1 & 3 & - & - & - & - & - & - \\
\hline Acquaintance/friend & 9 & 28 & 7 & 30 & 4 & 12 & 8 & 24 & 1 & 25 & - & - \\
\hline Stranger & - & - & - & - & 3 & 9 & 1 & 3 & - & - & - & - \\
\hline \multicolumn{13}{|l|}{ Weapon type } \\
\hline Other weapon & 10 & 31 & 11 & 48 & 10 & 30 & 9 & 27 & 1 & 25 & - & - \\
\hline Blunt instrument & 4 & 12 & 2 & 9 & 7 & 21 & 6 & 18 & 2 & 50 & - & - \\
\hline Personal weapons & 5 & 16 & 3 & 13 & 6 & 18 & 4 & 12 & 1 & 25 & 1 & 25 \\
\hline Firearms & - & - & - & - & 2 & 6 & 5 & 15 & - & - & - & - \\
\hline \multicolumn{13}{|l|}{ Location } \\
\hline House/apartment & 31 & 97 & 23 & 100 & 30 & 91 & 33 & 100 & 4 & 100 & 4 & 100 \\
\hline Transport area & - & - & - & - & 1 & 3 & - & - & - & - & - & - \\
\hline Commercial area & - & - & - & - & 1 & 3 & - & - & - & - & - & - \\
\hline Residential institution & - & - & - & - & 1 & 3 & - & - & - & - & - & - \\
\hline
\end{tabular}

*Presented figures are for Alaska, Maryland, Massachusetts, New Jersey, Oregon, South Carolina, and Virginia.

Columns may not add due to rounding.

homicides involving infants and young children 0-4 years of age.

Overall, $16 \%$ of the infant/child homicides involved blunt instruments. Within this particular weapon category, African American male infants/children accounted for $21 \%$ of homicide victims. African American females accounted for $18 \%$ of homicide victims in this weapon category. Twelve percent of white males and $9 \%$ of white females were victims of homicide via blunt instruments.

The relative risks for homicide using blunt instruments show that African American male infants/children were 6.0 times more likely to be slain by blunt instruments than white males (95\% CI 1.8 to 20.5 ), and 11.4 times more likely than white females (95\% CI 2.4 to 55.0). For African American females the risk of being slain by a blunt instrument was 5.0 times greater than the risk for their white female counterparts (95\% CI 1.5 to 189) and 10.1 times greater than that for white males (95\% CI 2.0 to 50.2). We found no significant differences in the rates of homicide via blunt instruments for African American males and females.

Personal weapons were used in $16 \%$ of all infant/child homicides. African American male infants/children accounted for $18 \%$ of these homicide victims. White male infants/children accounted for $16 \%$ of homicide victims via personal weapons followed by white females who accounted for $13 \%$ of homicide victims in this weapon category. Lastly, African American female infants/children accounted for $12 \%$ of homicide victims due to the use of personal weapons.

African American male infants/children were 4.1 times more likely to be murdered with personal weapons than white males (95\% CI 1.2 to 13.4). Moreover, African American male infants and young children were 6.5 times more likely to suffer homicide victimization via personal weapons than white females (95\% CI 1.4 to 26.1). Similarly, we found that the risk of being killed with a personal weapon among African American female infants and young children was 5.0 times greater than that of white females (95\% CI 1.0 to 20.1). We observed no significant differences in terms of the risks for homicide via personal weapons among African American males and females, nor for African American females when compared to white males.

Finally, NVDRS data indicate that firearms were used in only $5 \%$ of the homicides of children aged four years and under. However, it should be noted that African Americans infants and young children were again overrepresented as victims. In 2003-04, all firearms related infant/child homicide victims were African American.

\section{Multiple victims}

In $2003-04,71 \%$ of firearms related homicides of infants and young children involved multiple victims. Moreover, multiple victims were involved in $7 \%$ of homicides in which weapons designated as "other" were used. Further, multiple victims were involved in $4 \%$ of homicides where blunt instruments used and $44 \%$ of homicides involving a combination of weapons.

Three of these multiple victim incidents occurred within the context of intimate partner violence, three others occurred as the result of arguments, and one was precipitated by robbery. Arson was cited as the cause of death for one victim while another victim was murdered during an assault. The specific circumstances surrounding the deaths of four infants were unknown.

\section{Location and timing}

Homes and apartments including driveway, porch, or yard were the most frequent locations/places where infant/child homicide occurred. Public highways, streets, roads, as well as commercial, recreational, and natural areas were locations where at least one homicide occurred. Overall, 97\% of infant/ child homicides in 2003-04 occurred in a home/apartment. The percentage of deaths occurring in this location varied minimally across racial groups ranging from 91\% among African American male infants/children, to $100 \%$ for both African American and white female infants and young children.

Crude rate comparisons reveal that African American male infants and young children were 3.3 times more likely to be killed in homes or apartments than their white male counterparts (95\% CI 2.0 to 5.5). Moreover, African American male infants and young children were 4.3 times more likely to be killed in these locations than white female infants and young children (95\% CI 2.5 to 7.3). Similarly, for African American females aged four years and under, the probability of being murdered in a home or apartment was 3.8 times greater than that for white males (95\% CI 2.4 to 6.2) and 4.8 times more than that of white females (95\% CI 2.8 to 8.3 ). Lastly, prior studies have suggested that certain days of the week may be linked to a greater number of homicide occurrences among certain populations. ${ }^{14} 15$ 
However, we found no evidence of such a pattern for this age group.

\section{DISCUSSION}

\section{Implications for prevention}

Infant/child homicide is a serious issue. It is essential that the public health community continue to develop and refine prevention and intervention strategies aimed at curtailing the incidence and prevalence of this phenomenon. In order to develop appropriately targeted infanticide prevention and intervention programs, it is vital to have an informed knowledge of risk factors associated with this outcome. Such efforts may be bolstered by the use of information regarding the circumstances, events, and conditions related to infant/child homicide.

The current study identified the importance of the NVDRS as a surveillance tool, and why it should be considered in future analyses related to the study of child maltreatment in general and infant/child homicide in particular. The use of NVDRS data allowed for a broad examination of the circumstances, conditions, and patterns related to infant/ child homicide across all participating states. Few surveillance data are able to provide information pertaining to victim-suspect relationships, weapon type, multiple victims, location, and timing in the study of infant/child homicide. Hence, the NVDRS should continue to be developed and used to monitor patterns and possible trends related to infant/child homicide.

\section{Circumstances and characteristics}

NVDRS data indicate that African American infants and young children are at substantially greater risk for homicide victimization than are their white counterparts. Moreover, infant/child homicides were primarily perpetrated either by parents or friends and acquaintances and committed with weapons designated as "other". Lastly, the majority of these offences occurred in a home or an apartment. These findings corroborate prior research which asserts that infants and young children are most often murdered by those who are charged with, and/or responsible for their care and that homicides among this population are concentrated in primary care-giving environments. ${ }^{216}$

Within the context of the NVDRS, the designation "other" includes household items that because of close proximity or accessibility may be used as weapons of opportunity. This might indicate that a sudden loss of temper may play a noteworthy role in homicides among infants and young children. ${ }^{17}$ It is plausible that some individuals are simply unable to effectively cope with the demands and pressures of daily living and thus lash out in anger that unfortunately results in the death of a child. Certainly, the responsibility and care for young children can be extremely demanding. This, along with other duties, commitments, and responsibilities may over-tax individuals who have less than adequate coping resources, including lack of help-seeking behaviors and social support networks.

\section{High risk groups}

African Americans and other minorities are disproportionately exposed to marginal socioeconomic conditions. ${ }^{8}{ }^{10} 18$ Such conditions may compound the stressors associated with childcare, deplete internal coping reserves, and make it extremely difficult to access resources (for example, personal counseling services) that might reduce the likelihood of infant/child homicide. Some researchers have explored this supposition via the economic stress hypothesis which predicts maternal aggression and lethal violence in the context of economic stress. ${ }^{19}$ The comprehensive nature of the NVDRS makes it a prime vehicle by which to more thoroughly explore this hypothesis.

\section{Recommendations}

To reduce the potential for infant/child homicide, the public health community should use information like that provided by the NVDRS to identify emerging patterns and risk factors that may portend this most unfortunate outcome. Furthermore, in light of the racial disparities discussed herein, it is essential that the public health community develop prevention and intervention efforts that are sensitive to and reflective of the populations with whom they are to be used. $^{7}$

\section{Limitations}

The current study is subject to several limitations that can be attributed to the fluid nature of the NVDRS and the recent nature of its development and implementation. First, child abuse and maltreatment are widely acknowledged but largely untested risk factors for infant/child homicides. ${ }^{20}$ In the current investigation, the Child Fatality Review (CFR) records were examined in an effort to obtain additional information on the circumstances associated with infant/ child homicides. CFR records were available for only $13 \%$ $(n=9)$ of infant/child homicides in 2003 and $12 \%(n=7)$ of infant/child homicides in 2004. In those cases wherein a child fatality review was conducted, most contained responses of No, Unknown, Not available, Not reported, Not collected, or were missing variables that may have indicated a prior history of child maltreatment within the victim's household. These findings are due to the fact that the collection of CFR data is currently optional and also that only two states were funded to assess the availability and feasibility of collecting data from child fatality review teams. As a result, the CFR data cannot currently be used to extend our knowledge of the circumstances surrounding homicides among infants and young children. It is possible that the utility of this component of the NVDRS will increase substantially if CFR data are redefined as a primary NVDRS data element and subsequently collected more widely by NVDRS member states. The success of such an expansion would, nevertheless, hinge largely on availability (and standardization) of CFR data for all child deaths. The data currently available pertain largely to fatalities stemming from specific causes; the specific cause given attention may vary by state and by the data year of interest.

Moreover, the data did not contain information on household socioeconomic status when this study was conducted. Prior research has demonstrated a consistent link between socioeconomic status and rates of homicide. States with large numbers of impoverished women of childbearing age tend to have high rates of infant/child homicide. ${ }^{4} 18$ Ongoing efforts to expand the scope of the geospatial and economic data provided by the NVDRS will make it possible to eventually link NVDRS data to the zip codes of victims, suspects, and incident locations. This information could then be cross referenced with census tract data to obtain aggregate information pertaining to socioeconomic status. Such information may allow further exploration of the relationship between socioeconomic status and infant/child homicide.

Lastly, two final facts must be noted. First, while every effort is made to include all appropriate deaths, some might have been missed or not entered into the system when the present study was initiated. As a result it is suggested that the current research be considered an interim look at the NVDRS. Second, the presented rates and percentages only apply to the states used in the study's central analyses, although it is plausible that the results are more broadly applicable. 


\section{Key points}

- Homicides of young children are most often committed within the home, by parents or other caregivers.

- Weapons of opportunity were the most commonly used weapon type in the commission of infant/child homicide.

- African American infants and young children are at substantially greater risk for homicide victimization than are their white counterparts (4.2 times as great).

\section{Authors' affiliations}

M D Bennett Jr, J Hall, L Frazier Jr, L Barker, Etiology and Surveillance Branch, National Center for Injury Prevention and Control, Division of Violence Prevention, Centers for Disease Control and Prevention, Atlanta, GA, USA

N Patel, National Center for Injury Prevention and Control, Centers for Disease Control and Prevention, Office of Statistics and Programming, Atlanta, GA, USA

K Shaw, United Behavioral Health, Atlanta, GA, USA

The findings and conclusions in this paper are those of the authors and do not necessarily represent the views of the Centers for Disease Control and Prevention.

Competing interests: none.

\section{REFERENCES}

1 United States Department of Justice. Crime in the United States, 2003: Uniform Crime Reports. Washington, DC: Federal Bureau of Investigations, 2004.

2 Casey BM, Mclntire DD, Leveno KJ. The continuing value of the Apgar score for the assessment of newborn infants. Obstet Gynecol Surv 2001;56:406-7.
3 Kunz J, Bahr S. A profile of parental homicide against children. J Fam Violence 1996; 11:347-62.

4 Williams KR. Economic sources of homicide: Re-estimating the effects of poverty and inequality. Am Sociol Rev 1984;49:283-9.

5 Craig M. Perinatal risk factors for neonaticide and infant homicide" Can we identify those at risk? J R Soc Med 2004;97:57-61.

6 Cubbin C, LeClere FB, Smith GS. Socioeconomic disadvantage and injury mortality: Individual and neighbourhood determinants. J Epidemiol Community Health 2000;54:517-24.

7 Gauthier D, Chaudoir NK, Forsyth CJ. A sociological analysis of maternal infanticide in the United States. Deviant Behav 2003;24:393-404.

8 Herman-Giddens ME, Smith JB, Mittal M, et al. Newborns killed or left to die by a parent: A population based study. Obstet Gynecol Surv 2003;58:580-81

9 Overpeck MD, Brenner RA, Trumble AC, et al. Risk factors for infant homicide in the United States. N Engl J Med 1998;339:1211-16.

10 Ingram DD, Parker JD, Schenker N, et al. United States Census 2000 population with bridged race categories. National Center for Health Statistics. Vital Health Stat 2003;135: 1-55.

11 Jain A, Khoshnood B, Lee K, et al. Injury related infant death: The impact of race and birth weight. Inj Prev 2001;7:135-40.

12 Centers for Disease Control and Prevention, National Center for Injury Prevention and Control. Web-based Injury Statistics Query and Reporting System (WISQARS). Available at http://www.cdc.gov/ncipc/wisqars (accessed February 2006).

13 Agresti A, Coull B. Approximate is better than exact for interval estimation of binomial proportions. Am Stat 1998;52:119-26.

14 Apgar V. A proposal for a new method of evaluation of the newborn infant. Curr Res Anesth Analg 1953;32:260-7.

15 McCleary R, Chew KS. Winter is the infanticide Season: Seasonal risk for child homicide. Homicide Studies 2002;6:228-39.

16 Greenberg M, Schneider D. Blue Thursday? Homicide and suicide among urban 15-24-year-old black male Americans. Public Health Rep 1992; 107:264-8.

17 Overpeck MD, Brenner RA, Trumble AC, et al. Infant injury deaths with unknown intent: What else do we know? Inj Prev 1999:5:272-5.

18 Paulozzi L, Sells M. Variation in homicide risk during infancy-United States 1989-1998. MMWR Morb Mortal Wkly Rep 2002;51:187-9.

19 Phipps MG, Sowers M, Demonner SM. The risk for infant mortality among adolescent childbearing groups. J Womens Health 2002;11:889-97.

20 Sorenson SB, Peterson JG. Traumatic child death and documented maltreatment history, Los Angeles. Am J Public Health 1994;84:623-7. 